\title{
Uniform Cluster Estimates for Lattice Models
}

\author{
V. A. Malyshev \\ Moscow State University, 117234 Moscow B-234, USSR
}

\begin{abstract}
In [2] we obtained the complete cluster expansion for transfermatrix for lattice models with bounded potentials in high-temperature region. Here we obtain necessary cluster estimates for semiinvariants of functionals over Gibbs field with unbounded interaction in high-temperature region and for contour models in low-temperature region.
\end{abstract}

\section{Contents}

Introduction . . . . . . . . . . . . . . . . . . . . . . . . . . . . . . . . . . . 131

Part 1. Auxiliary bounds . . . . . . . . . . . . . . . . . . . . . . . . . . . 132

1.1. Connectivity bounds . . . . . . . . . . . . . . . . . . . . . . . . . . . . . 132

1.2. Bounds on semiinvariants of independent virtual field . . . . . . . . . . . . . . . 133

1.3. Bounds on the intersection numbers . . . . . . . . . . . . . . . . . . . . . . . 137

Appendix to Part 1. Calculation of Möbius functions for lattices . . . . . . . . . . . . . 141

Part 2 . . . . . . . . . . . . . . . . . . . . . . . . . . . . . . . 147

2.1. The main result . . . . . . . . . . . . . . . . . . . . . . . . . . . . . . . 147

2.2. High-temperature expansions . . . . . . . . . . . . . . . . . . . . . . . . . 151

2.3. Contour cluster expansions for sufficiently low temperatures . . . . . . . . . . . . 154

\section{Introduction}

One of the main obstacles on the way to the proof of asymptotic completeness for $\lambda P(\varphi)_{2}$ models of quantum field theory for small $\lambda$ was the absence of $N$-particle cluster expansion for all $N$ in $0<\lambda<\lambda_{0}$. Glimm, Jaffe and Spencer [1] obtained $N$-particle cluster expansion with $N \rightarrow \infty$ if $\lambda \rightarrow 0$.

In [2] the author obtained the complete cluster expansion (i.e. for all $N$ ) in high temperature region $|\beta|<\beta_{0}$ for lattice fields with bounded interaction potential. The main ingredients in [2] were strong cluster estimates of semiinvariants $\left\langle F_{B_{1}}, \ldots, F_{B_{n}}\right\rangle$ where $F_{B}$ depends only on values of Gibbs random field in points $\llcorner B, B$ is finite. 
If e.g. $\left|F_{B_{i}}\right| \leqq 1$ then these estimates are

$$
\left\langle F_{B_{1}}, \ldots, F_{B_{n}}\right\rangle \mid \leqq(C \beta)^{d\left(B_{1}, \ldots, B_{n}\right)} \prod_{i=1}^{n} u_{i} C^{d_{B_{i}}}
$$

where $d\left(B_{1}, \ldots, B_{n}\right)$ is roughly the smallest length of a connected tree with $n$ vertices $b_{i} \in B_{i} ; d_{B}$ is the smallest length of a connected tree with vertices in $B$ and $u_{i}$ is the number of $j=1, \ldots, n$ such that $B_{i}=B_{j}$.

We obtain here estimates $(0.1)$ for the case of unbounded potential in hightemperature region and for contour functionals for the Ising model in low temperature region. We should emphasize that our estimates are valid uniformly in some region of temperature for arbitrary $B_{1}, \ldots, B_{n}$.

Nonuniform estimates with restriction to the size of $B_{1}, \ldots, B_{n}$ can be obtained by analytical methods of $[3,4]$ and by method of [5].

In some other place we shall derive from this uniform $N$-particle cluster expansion for transfer matrix for these models.

Our methods seem to be applicable to models of quantum field theory also.

Our strategy is the following. We use correlation equations (of KirkwoodSalsburg or Minlos-Sinai type) to get an explicit series for moments of Gibbs random fields. We use this series to get explicit series for semiinvariants. In the latter series we perform cancellations using Möbius function theory (we use one theorem of J.-C. Rota) and some other auxiliary bounds.

To make this paper selfcontained we include all results we need from Möbius function theory.

We prove cluster estimate $(0.1)$ first in abstract situation $(\$ 2.1)$ and prove then that this situation takes place in high-temperature (\$2.2) and low-temperature (§2.3) region.

\section{Part 1. Auxiliary Bounds}

\subsection{Connectivity}

This paragraph contains mainly definitions and simple lemmas.

A set $A \subset \mathbb{Z}^{v}$ is called $d$-connected it for each two points $t, t^{\prime} \in A$ there exists a sequence $t=t_{1}, t_{2}, \ldots, t_{n}=t^{\prime}$ of points $t_{i} \in A$ such that $\left|t_{i}-t_{i-1}\right| \leqq d$ for $i=2, \ldots, n$.

A collection $\Gamma=\left(A_{1}, \ldots, A_{n}\right)$ of sets $A_{i} \subset \mathbb{Z}^{v}$ is called connected if the following graph $G_{\Gamma}$ is connected : it has vertices $1, \ldots, n$ and there is a line connecting $i$ and $j$ iff $A_{i} \cap A_{j} \neq \emptyset$.

Lemma 1. There is a constant $C=C(v, d)$ such that the number of (different) $d$-connected sets $A$ of cardinality $r$ containing some fixed point $t$ does not exceed $C^{r}$.

Lemma 2. There is a constant $C=C(v, \varrho)$ such that the number of connected collections $\Gamma=\left(A_{1}, \ldots, A_{n}\right)$ containing some fixed point $t$ in which all $A_{i}$ are pairwise different and diam $A_{i} \leqq \varrho$ does not exceed $C^{n}$.

Further we shall often consider systems $\mathfrak{A}$ of finite subsets of $\mathbb{Z}^{v}$. We shall always assume that $\mathfrak{A}$ is connected (i.e. graph $G_{\mathfrak{O}}$ is connected) translation invariant and diameter of elements of $\mathfrak{U}$ is uniformly bounded. 
For any finite $A \subset \mathbb{Z}^{v}$ and any collection $\alpha$ of finite subsets $B_{i} \subset \mathbb{Z}^{v}$ we denote: $d_{A}$, minimal $d$ such that there exists 1 -connected set $B$ containing $A$ and $|B|=d$; $d_{A}(\mathfrak{U})\left(\delta_{A}^{\alpha}(\mathfrak{U})\right)$, minimal $d$ such that there exists connected (respectively, such that collection $\Gamma \cup \alpha$ is connected) collection $\Gamma=\left(A_{1}, \ldots, A_{d}\right)$ such that $A_{i} \in \mathfrak{A}$ and

$$
\tilde{\Gamma} \equiv \bigcup_{i=1}^{d} A_{i} \supset A
$$

Agreement About Constants. Further $C, C_{1}, C_{2}, \ldots$ will denote constants which depend only on dimension $v$ of $\mathbb{Z}^{v}$ and possibly on system $\mathfrak{A}$ in question. In one formula one letter, e.g. $C$, can denote different numbers.

All these constants can be easily calculated.

It is not difficult to show that

$$
C_{1} d_{A} \leqq d_{A}(\mathfrak{U}) \leqq C_{2} d_{A} \text {. }
$$

Lemma 3. The number of subsets $B \subset \mathbb{Z}^{v}$ containing fixed point $t$ and such that $d_{B}=r$ does not exceed $C^{r}$.

Proof. Let $B_{1}, \ldots, B_{p}$ - all these subsets. Let $D_{i}$ be 1-connected, $D_{i} \supset B_{i}$ and $\left|D_{i}\right|=d_{B_{i}}=r$. For each $i$ there may be not more than $2^{r}$ subsets among $D_{1}, \ldots, D_{p}$ coinciding with $D_{i}$ (as if $D_{i}=D$; then $B_{j} \subset D_{i}$ and there is not more than $2^{r}$ subsets of $D_{i}$ ).

Lemma 4. For each $C$ there exists $C_{1}$ such that for each finite $T \subset \mathbb{Z}^{v}$ and for $\lambda>0$ sufficiently small

$$
\sum_{\Gamma}(C \lambda)^{|\Gamma|} \leqq\left(C_{1} \lambda\right)^{n} C_{2}|T|
$$

where the summation is through all $\Gamma$ such that $|\Gamma| \geqq n$ and collection $(\Gamma, T)$ is connected (compare with Lemma 6.2 of [2]).

\subsection{Bounds on Semiinvariants of Independent Virtual Field}

Let a set $V$ be given. Let for any nonempty finite $A \subset V$ be given a number $f(A)$. A collection of numbers $f(A)$ we shall consider as moments of virtual field to justify the following standard definition of formal semiinvariants $g(A)$ of this virtual field by induction

$$
f(A)=\sum g\left(B_{1}\right) \ldots g\left(B_{k}\right)
$$

where the summation is through all (non-ordered) partitions $B_{1} \cup B_{2} \cup \ldots \cup B_{k}$ of the set $A$.

We define for all partitions $\alpha=\left(A_{1}, \ldots, A_{k}\right)$ of the set $V$.

$$
f(\alpha)=\prod_{i=1}^{k} f\left(A_{i}\right), \quad g(\alpha)=\prod_{i=1}^{k} g\left(A_{i}\right) .
$$

Let $\mathfrak{A}$ be the lattice of all partitions of the set $V$ (see Appendix). From Möbius inversion formula due to

$$
f(\alpha)=\sum_{\beta \leqq \alpha} g(\beta)
$$


one has

$$
g(\alpha)=\sum_{\beta \leqq \alpha} \mu_{\mathfrak{Q}}(\beta, \alpha) f(\beta)
$$

(The explicit expression for $\mu_{\mathfrak{2}}(\beta, \alpha)$ is well-known and proved e.g. in [6].)

Let now graph $G$ be given the set of vertices of which is $V$. (For all graphs considered below we assume that there is not more than one line between each pair of vertices.) Let us denote $G_{A}, A \subset V$, a subgraph of $G$ with $A$ as a set of its vertices. We shall call $G_{B}$ a component of $G_{A}$ iff there is no lines between $B$ and $A-B$.

Let now function $f(A)$ enjoy the following remarkable property

$$
f(A)=f\left(A_{1}\right) \ldots f\left(A_{k}\right)
$$

if for all $i G_{A_{\imath}}$ is a component of $G_{A}$.

Such virtual field $f(A)$ we call independent with respect to $G$.

Lemma 1. If $G_{A}$ is not connected and property (5) takes place then

$$
g(A)=0 \text {. }
$$

Proof. By induction in $|A|$

$$
g(A)=f(A)-\sum^{\prime} g\left(B_{1}\right) \ldots g\left(B_{k}\right)
$$

where the summation in $\sum^{\prime}$ is through all partitions of $A$ except trivial one.

Let $A_{1}, \ldots, A_{p}$ - all connected components of $A$ (i.e. $G_{A_{2}}$ are connected components in $G_{A}$ ).

Then by induction $g\left(B_{i}\right)$ can be different from 0 only if $B_{i}$ belongs to some $A_{j}$. From (7)

$$
g(A)=f(A)-\sum^{\prime} f\left(A_{2}\right) \ldots f\left(A_{p}\right)=0 .
$$

Let us denote

$$
C_{f}(A)=\max \left|\prod_{i=1}^{k} f\left(B_{i}\right)\right|
$$

where maximum is through all partitions $\left(B_{1}, \ldots, B_{k}\right)$ of $A$.

Theorem 1. Let $G_{A}$ be connected. Then

$$
|g(A)| \leqq \frac{3}{2} C_{f}(A) \prod_{t \in A} 3 v_{t}
$$

where $v_{t}=v_{t}^{(A)}$ is the number of lines in $G_{A}$ incident with the vertex $t \in A$.

To prove this theorem we remark that due to Lemma 1 we can rewrite (4) as

$$
f(\alpha)=\sum_{\beta: \beta \leqq \alpha, \beta \in \mathfrak{Q}_{G}} g(\beta) .
$$

If we restrict (10) to $\alpha \in \mathfrak{A}_{G}$ then we can use Möbius inversion formula for $\mathfrak{A}_{G}$ to get for $\alpha \in \mathfrak{U}_{G}$

$$
g(\alpha)=\sum_{\beta \leqq \alpha, \beta \in \mathfrak{A}_{G}} f(\beta) \mu_{\mathfrak{R}_{G}}(\beta, \alpha) .
$$




\section{Lemma 2.}

$$
\left|\mu_{\mathfrak{M}_{G}}(0,1)\right| \leqq \prod_{t \in V} v_{t}
$$

where $v_{t}$ is the number of lines in $G$ incident with vertex $t \in V$.

Proof. We shall use Corollary A.1 where $|V|=n$. Then

$$
\left|\mu_{\mathfrak{Q}_{G}}(0,1)\right| \leqq m_{1} \leqq \tilde{m}_{1}
$$

where $\tilde{m}_{1}$ is the number of subsets $X$ of lines of $G$ such that $|X|=n-1$ and $X$ does not contain any circuit.

To bound $\tilde{m}_{1}$ we remark first that $X$ must be connected tree: $X$ is a tree as it does not contain any circuit and $X$ is connected as $|X|=n-1$.

We shall define now one-to-one map $\varphi_{X}$ of the set $X$ onto some $A \subset V$ with $|A|=n-1$. For this reason let us consider any $\gamma_{1} \in X$ and define $\varphi_{X}\left(\gamma_{1}\right)$ to be any of two vertices incident with $\gamma_{1}$. Let us suppose by induction that we have already defined $\gamma_{1}, \ldots, \gamma_{m} \in X$ and $\varphi_{X}\left(\gamma_{1}\right), \ldots, \varphi_{X}\left(\gamma_{m}\right)$. Let $V_{m}$ be a set of vertices incident with some of $\gamma_{1}, \ldots, \gamma_{m}$. Let us choose a line $\gamma_{m+1}$ such that one of its vertices belongs to $V_{m} \cdot \gamma_{m+1}$ exists - otherwise a set $\left\{\gamma_{1}, \ldots, \gamma_{m}\right\}$ must contain a circuit. Moreover both vertices of $\gamma_{m+1}$ cannot belong to $V_{m}$ - otherwise $\left\{\gamma_{1}, \ldots, \gamma_{m+1}\right\}$ contains a circuit as $\left\{\gamma_{1}, \ldots, \gamma_{m}\right\}$ connected by this inductive construction.

We put $\varphi_{X}\left(\gamma_{m+1}\right)$ to be that of two vertices of $\gamma_{m+1}$ which belongs to $V_{m}$ if it does not coincide with one of earlier defined vertices $\varphi_{X}\left(\gamma_{1}\right), \ldots, \varphi_{X}\left(\gamma_{m}\right)$ and other of these two vertices in the opposite case.

We define now a map $\psi_{X}$ of the set $V$ into the set of lines of $G$ putting $\psi_{X}(v)=\gamma_{i}$ if $\varphi_{X}\left(\gamma_{i}\right)=v, v \in V$, and if there is no such $\gamma_{i}$ then we put $\psi_{X}(v)$ equal to any of $\gamma_{i}$ incident to $v$. All $\psi_{X}$ are different as $X$ are different. So, the number of all such $X$ does not exceed the number of all maps from $V$ to the set of lines mapping each $v \in V$ on a line incident to it. The number of such maps is equal to $\prod_{t \in V} v_{t}$.

Lemma is proved.

Theorem will evidently follow from (11) if we shall prove

\section{Lemma 3.}

$$
\sum_{\beta \in \mathfrak{A}_{G}}\left|\mu_{\mathfrak{A}_{G}}(\beta, 1)\right| \leqq \frac{3}{2} \prod_{t \in V} 3 v_{t}
$$

To prove this Lemma we note that

$$
\mu_{\mathfrak{P}_{G}}(\beta, 1)=\mu_{\mathfrak{Q}_{G(\beta)}}(0,1)
$$

where $G(\beta)$ is the graph obtained from the graph $G$ in the following way: we identify all vertices belonging to the same block $B_{i}$ of partition $\beta$, i.e. vertices of $G(\beta)$ are blocks of $\beta$. Two new vertices $B_{i}$ and $B_{j}$ are connected with a line iff there is a line in $G$ between some $b_{1} \in B_{1}$ and $b_{2} \in B_{2}$. So

$$
\mu_{\mathfrak{Q}_{G(\beta)}}(0,1)=\mu_{[\beta, 1]}(\beta, 1)=\mu_{\mathfrak{Q}_{G}}(\beta, 1)
$$

due to property (D5). 
Proof of Lemma 3. We shall construct inductively a tree $\mathbf{6}$. Rules for unique specification of this tree are the following.

1. Vertices of $\mathfrak{5}$ have other $1,2, \ldots, n$ and each vertex is coloured in red or blue.

2. To each vertex corresponds exactly one graph $G\left(\beta_{t}\right)$, subset $D_{t}$ of vertices of $G\left(\beta_{t}\right)$ and $\beta_{t} \in \mathfrak{H}_{G}$.

3. Vertex of order 1 is only one. It is red. Corresponding to her are $G\left(\beta_{t}\right)=G$ and $D_{t}=\emptyset$. This vertex is the highest vertex of the tree in question.

4. Over each vertex of order $K(K$-vertex $)$ there is exactly one $(K-1)$-vertex.

5 . Let $K$-vertex $t$ is already constructed together with $G\left(\beta_{t}\right)$ and $D_{t}$ corresponding to her.

Let $v_{t}(\xi)$ - the number of lines of $G\left(\beta_{t}\right)$ incident with vertex $\xi$ of $G\left(\beta_{t}\right)$. Let $\xi_{1}, \ldots, \xi_{m}-$ all vertices of $G\left(\beta_{t}\right)$ not belonging to $D_{t}$ and moreover

$$
v_{t}\left(\xi_{1}\right) \leqq v_{t}\left(\xi_{2}\right) \ldots \leqq v_{t}\left(\xi_{m}\right)
$$

Under $t$ we shall construct not more than $v_{t}\left(\xi_{1}\right)$ red $(K+1)$-vertices and not more than one blue vertex.

This blue vertex will have the same $G\left(\beta_{t}\right)$ as for $t$ but to $D_{t}$ we add vertex $\xi_{1}$ (if $m \neq 0$ ). If $m=0$ we do not construct blue vertex.

From $G\left(\beta_{t}\right)$ one can construct a new graph $G\left(\beta_{t^{\prime}}\right)$ in not more that $v_{t}\left(\xi_{1}\right)$ ways if one will identify vertex $\xi_{1}$ with one of other vertices connected with $\xi_{1}$ with a line and not belonging to $D_{t}$.

Each such $G\left(\beta_{t^{\prime}}\right)$ will correspond to a new red $(K+1)$-vertex. $D_{t^{\prime}}$ for this vertex is the same as for $t$ (it is correct as we do not identify vertices of $D_{t}$ in construction of $G\left(\beta_{t^{\prime}}\right)$.

Proposition 1. For each $\beta \in \mathfrak{U}_{G}$ there exists red $t \in \mathfrak{G}$ such that $\beta=\beta_{t}$.

Proof. Let $v(1) \leqq v(2) \leqq \ldots \leqq v(n)$ for vertices $1, \ldots, n$ of $G$.

1 st case. $\{1\}$ forms a separate block of $\beta$. Then we construct a blue vertex $t$ of order 2 with $\beta_{t}=0$ and corresponding $D_{t}$ has one vertex.

2 nd case (opposite to $1 \mathrm{st}$ ). In construction red vertices of order 2 let us choose either of them under the condition that this vertex $t$ will have $\beta_{t} \leqq \beta$ and $D_{t}=\emptyset$.

So we are reduced to the case when graph has one vertex less. Then we proceed by induction.

Let us put for each $t \in \mathfrak{G}$

$\gamma_{t}=\prod_{\xi \in G\left(\beta_{t}\right)} v_{t}(\xi)$

Let $K$-vertex $t$ be given and $(K+1)$-vertex $t^{\prime}$ under $t$.

If $t^{\prime}$ is blue then by construction

$\gamma_{t}=\gamma_{t^{\prime}}$

If $t^{\prime}$ is red then

$$
\gamma_{t^{\prime}} / \gamma_{t} \leqq \frac{v_{t}\left(\xi_{1}\right)+v_{t}\left(\xi_{r}\right)-2}{v_{t}\left(\xi_{1}\right) v_{t}\left(\xi_{r}\right)} \leqq \frac{2}{v_{t}\left(\xi_{1}\right)}
$$


From (16) we get

$$
\sum \gamma_{t^{\prime}} \leqq v_{1} \frac{2}{v_{1}} \gamma_{t} \leqq 2 \gamma_{t}
$$

where the summation is through all red $(K+1)$-vertices $t^{\prime}$ under $t$.

Consequently

$$
\begin{aligned}
& \sum_{\begin{array}{c}
\text { red } t \\
\text { of order } K+1
\end{array}} \gamma_{t} \leqq 2 \sum_{\substack{\text { red } t \\
\text { of order } K}} \gamma_{t}+2 \sum_{\begin{array}{c}
\text { blue } t \\
\text { of order } K
\end{array}} \gamma_{t} \\
& \leqq 2 \sum_{\substack{\text { red } t \\
\text { of order } K}} \gamma_{t}+2 \sum_{\begin{array}{c}
\text { red } t \\
\text { of order } K-1
\end{array}} \gamma_{t}+2 \sum_{\begin{array}{c}
\text { blue } t \\
\text { of order } K-1
\end{array}} \gamma_{t} \\
& \leqq \ldots \leqq 2 \sum_{\begin{array}{c}
\text { red } t \\
\text { of order } \leqq K
\end{array}} \gamma_{t} .
\end{aligned}
$$

Put

$$
a_{K}=\sum_{\substack{\text { red } t \\ \text { of order } K}} \gamma_{t} .
$$

We have

$$
a_{K+1} \leqq 2 \sum_{i=1}^{K} a_{i}
$$

from where it follows that $a_{K} \leqq 3^{K} a_{1}$.

By Proposition 1, Lemma 2 and (14)

$$
\sum_{\beta \in \mathfrak{R}_{G}}|\mu(\beta, 1)| \leqq \sum_{\operatorname{red} t} \gamma_{t} \leqq a_{1} \sum_{K=1}^{n} 3^{K} \leqq \frac{3}{2} \prod_{i=1}^{n} 3 v_{i} \text {. }
$$

Lemma 3 is proved.

\subsection{Bounds on the Intersection Numbers}

Let a collection $\alpha$ of finite sets $A_{1}, \ldots, A_{n} \subset \mathbb{Z}^{v}$ be given (some of $A_{i}$ can coincide). We define

$$
\begin{aligned}
& v_{i}=v_{i}(\alpha), \text { number of } A_{j}, j=1, \ldots, n, \text { such that } A_{i} \cap A_{j} \neq \emptyset ; \\
& u_{i}=u_{i}(\alpha), \text { number of } A_{j}, j=1, \ldots, n, \text { such that } A_{i}=A_{j} .
\end{aligned}
$$

Theorem 1. There exists a constant $C>0$ depending only upon $v$ and such that for each $N$ and each system $\alpha=\left(A_{1}, \ldots, A_{n}\right)$ of 1 -connected finite subsets of $\mathbb{Z}^{v}$

$$
C \sum_{i=1}^{N}\left|A_{i}\right|+\sum_{i=1}^{N} \ln u_{i} \geqq \sum_{i=1}^{N} \ln v_{i} .
$$

Proof. It is convenient to use the following notations. Let us divide $\alpha$ onto equivalence classes: $A_{i}$ and $A_{j}$ are equivalent iff they coincide as sets. The set of all equivalence classes we denote $\mathscr{A}$. Other notations: $\mathscr{A}_{k}$, the set of all equivalence classes of power $k$;

$\mathscr{A}^{r}$, the set of all equivalence classes such that if $A_{i}$ belong to such equivalence class then $|A|=r$;

$\mathscr{A}_{k}^{r}$, the set of equivalence classes with $k$ elements each of which has power $r$. We put

$$
\alpha_{k}=\left|\mathscr{A}_{k}\right|, \quad \alpha^{r}=\left|\mathscr{A}^{r}\right|, \quad \alpha_{k}^{r}=\left|\mathscr{A}_{k}^{r}\right| \text {. }
$$


Let $\omega_{k r ; k^{\prime} r^{\prime}}$ be the number of pairs $\left(\chi, \chi^{\prime}\right), \chi \in \mathscr{A}_{k}^{r}, \chi^{\prime} \in \mathscr{A}_{k^{\prime}}^{r^{\prime}}$, such that (each) representative of class $\chi$ has nonempty intersection with (each) representative of class $\chi^{\prime}$.

We shall use the following evident properties of these numbers:

1. (symmetry) $\alpha_{k r ; k^{\prime} r^{\prime}}=\alpha_{k^{\prime} r^{\prime} ; k r}$,

2. (normalization) $\sum_{k} k \alpha_{k}=\sum_{k, r} k \alpha_{k r}=N$.

3. Let $A_{i}$ be fixed. Then the number of classes in $\mathscr{A}_{r}$ the elements of which has nonempty intersection with $A_{i}$ does not exceed

$\left|A_{i}\right| C^{r}$.

This follows from Lemma 1, 1.1.

We can rewrite (1) now

$$
C \sum_{k, r} r k \alpha_{k r}+\sum_{k, r} k \alpha_{k r} \ln k \geqq \sum_{i=1}^{N} \ln v_{i} .
$$

We have from definitions

$$
\sum_{i: \mathscr{A}_{k}^{r}} v_{i}=k \sum_{k^{\prime}, r^{\prime}} \alpha_{k r ; k^{\prime} r^{\prime}} k^{\prime}
$$

where the summation in $\sum_{i: \mathscr{A}_{\mathrm{k}}^{r}}$ is through all $i$ such that $A_{i}$ is a representative of a class belonging to $\mathscr{A}_{k}^{r}$. It follows that

$$
\sum_{i: \mathscr{A}_{k}^{r}} \ln v_{i} \leqq k \alpha_{k r} \ln \frac{k \sum_{k^{\prime}, r^{\prime}} \alpha_{k r ; k^{\prime} r^{\prime}} k^{\prime}}{k \alpha_{k r}} .
$$

Here we used inequality

$$
\gamma_{1} \ldots \gamma_{k} \leqq\left(\frac{\gamma}{k}\right)^{k}
$$

for all $\gamma_{1}, \ldots, \gamma_{k}>0$ such that $\gamma_{1}+\ldots+\gamma_{k}=\gamma, \gamma>0$.

Due to (4) it is sufficient to prove that

$$
C d N+\sum_{k, r} k \alpha_{k r} \ln k \alpha_{k r} \geqq \sum_{k, r} k \alpha_{k r} \ln \beta_{k r}
$$

where

$$
\beta_{k r}=\sum_{k^{\prime}, r^{\prime}} \alpha_{k r ; k^{\prime} r^{\prime}} k^{\prime}
$$

and

$$
d=\frac{1}{N} \sum_{i=1}^{N}\left|A_{i}\right|
$$

is the mean power for system $A_{1}, \ldots, A_{N}$.

From property 3 it follows that

$$
\sum_{k} \alpha_{k r ; k^{\prime} r^{\prime}} \leqq r^{\prime} C^{r} \alpha_{k^{\prime}}^{r^{\prime}}
$$


and so

$$
\begin{aligned}
\sum_{k} \beta_{k r} & =\sum_{k^{\prime}, r^{\prime}} k^{\prime} \sum_{k} \alpha_{k r ; k^{\prime} r^{\prime}} \\
& \leqq \sum_{k^{\prime}, r^{\prime}} k^{\prime} r^{\prime} C^{r} \alpha_{k^{\prime}}^{r^{\prime}}=C^{r} d N .
\end{aligned}
$$

We denote $\delta_{k r}=k \alpha_{k}^{r}$ and shall prove that

$$
C d N+\sum_{k, r} \delta_{k r} \ln \delta_{k r} \geqq \sum_{k, r} \delta_{k r} \ln \beta_{k r}
$$

under the condition (7).

For fixed $r$ and $\delta_{k r}$

$$
\sum_{k} \delta_{k r} \ln \beta_{k r}
$$

attaches its maximum value when $\beta_{k r}$ are proportional to $\delta_{k r}$.

It is sufficient to prove this assertion for the case

$$
\delta_{k_{1} r} \ln \beta_{k_{1} r}+\delta_{k_{2} r} \ln \beta_{k_{2} r}
$$

with sum $\beta=\beta_{k_{1} r}+\beta_{k_{2} r}$ fixed and fixed $\delta_{k_{2} r}$. But it is a result of simple calculation.

It follows from this assertion that

$$
\begin{aligned}
\sum_{k} \delta_{k r} \ln \beta_{k r} & \leqq \sum_{k} \delta_{k r} \ln \delta_{k r} \frac{\sum_{k} \beta_{k r}}{\sum_{k} \delta_{k r}} \\
& \leqq \sum_{k} \delta_{k r} \ln \frac{\delta_{k r}^{k} C^{r} d N}{\delta_{r}} .
\end{aligned}
$$

Due to (9) it is sufficient to prove that

$$
C d N+\sum_{k, r} \delta_{k r} \ln \delta_{k r} \geqq \sum_{k, r} \delta_{k r} \ln \frac{\delta_{k r} C^{r} d N}{\delta_{r}}
$$

or equivalently that

$$
C d \geqq-\sum_{r} \tilde{\delta}_{r} \ln \tilde{\delta}_{r}
$$

where $\tilde{\delta}_{r}=\delta_{r} / N$ under the conditions

$$
\sum \tilde{\delta}_{r}=1, \quad \sum r \tilde{\delta}_{r}=d .
$$

But

$$
-\sum_{r \leqq e^{d}} \tilde{\delta}_{r} \ln \tilde{\delta}_{r} \leqq \ln e^{d}=d
$$

is a well-known property of entropy.

At the same time

$$
-\sum_{r>e^{d}} \tilde{\delta}_{r} \ln \tilde{\delta}_{r} \leqq \sum_{r>e^{d}} r \tilde{\delta}_{r}+\sum_{r>e^{d}} \frac{r}{e^{r}} \leqq 2 d .
$$


The first inequality in (13) is derived from the alternative: either

$\tilde{\delta}_{r} \geqq e^{-r}$

and then

$$
\text { or } \begin{aligned}
& -\tilde{\delta}_{r} \ln \tilde{\delta}_{r} \leqq \tilde{\delta}_{r} r \\
& \delta_{r}<e^{-r}
\end{aligned}
$$

and then

$$
-\tilde{\delta}_{r} \ln \tilde{\delta}_{r} \leqq \frac{r}{e^{r}}
$$

(as $x / e^{x}$ is monotone decreasing in $x$ ).

Then (11) follows from (12) and (13).

Theorem is proved.

Theorem 2. There exist $C>0$ such that for each system $A_{1}, \ldots, A_{N}$

$$
C \sum_{i=1}^{N} d_{A_{i}}+\sum_{i=1}^{N} \ln u_{i} \geqq \sum_{i=1}^{N} \ln v_{i} .
$$

Proof. One can follow most closely the proof of Theorem 1 with the following modifications: $\mathscr{A}^{r}$ will denote a set of all classes each representative $A_{i}$ of which has $d_{A_{i}}=r$. Property 3 follows from Lemma 3,1.1. Theorem is proved.

Let now be given a system $\mathscr{A}$ consisting of $N$ not necessary different finite subsets $A_{1}, \ldots, A_{N} \subset \mathbb{Z}^{v}$ and let be given also $m$ pairwise different points $t_{1}, \ldots, t_{m} \in \mathbb{Z}^{v}$.

We divide system $\mathscr{A}$ on classes in two different ways. First partition $\alpha_{1}, \ldots, \alpha_{n}$ is such that

$$
\alpha_{1} \cup \ldots \cup \alpha_{n}=\mathscr{A}, \quad \alpha_{i} \cap \alpha_{j}=\emptyset \quad \text { if } \quad i \neq j
$$

and all $A_{j}$ belonging to one $\alpha_{i}$ are the same but if $A_{i}$ and $A_{j}$ belong to different classes they are necessarily different.

The second partition $\delta_{1}, \ldots, \delta_{m}$ has the following property: for all $i$ each $A_{k} \in \delta_{i}$ contains point $t_{i}$.

(The second partition is not uniquely determined by this condition. We consider arbitrary such partition assuming existence.)

Lemma 1. In these notations

$$
\sum_{i=1}^{n}\left|\alpha_{i}\right| \ln \left|\alpha_{i}\right| \leqq \sum_{i=1}^{m}\left|\delta_{i}\right| \ln \left|\delta_{i}\right|+\sum_{i=1}^{N}\left|A_{i}\right|
$$

Proof. Put

$$
k_{i j}=\left|\alpha_{i} \cap \delta_{j}\right| .
$$

Then

$$
\sum_{j=1}^{m}\left|\delta_{j}\right| \ln \left|\delta_{j}\right| \geqq \sum_{i, j} k_{i j} \ln k_{i j} .
$$


Let us consider class $\alpha_{i}$. Let there exist exactly $r$ classes $\delta_{i_{1}}, \ldots, \delta_{j_{r}}$ such that $\alpha_{i} \cap \delta_{j_{1}} \neq \emptyset, \ldots, \alpha_{i} \cap \delta_{j_{r}} \neq \emptyset$.

Then for

$$
A \in \alpha_{i}, \quad|A| \geqq r .
$$

As

$$
\sum_{p=1}^{r} k_{i j_{p}}=\left|\alpha_{i}\right|
$$

then by entropy inequality

$$
\sum_{j} k_{i j} \ln k_{i j} \geqq \frac{r\left|\alpha_{i}\right|}{r} \ln \frac{\left|\alpha_{i}\right|}{r}=\left|\alpha_{i}\right| \ln \frac{\left|\alpha_{i}\right|}{r}
$$

As

$$
\sum_{A \in \alpha_{i}}|A| \geqq\left|\alpha_{i}\right| r
$$

then

$$
\sum_{A \in \alpha_{i}}|A|+\sum_{j} k_{i j} \ln k_{i j} \geqq\left|\alpha_{i}\right| \ln \left|\alpha_{i}\right|
$$

From (17) and (16) we have (15).

From this lemma and from Theorem 1 we get

Corollary 1. Let $N$ 1-connected (not necessarily different) finite subsets $A_{1}, \ldots, A_{N} \subset \mathbb{Z}^{v}$ and $m$ pairwise different points $t_{1}, \ldots, t_{m} \in \mathbb{Z}^{v}$ be given. Then

$$
C \sum_{i=1}^{N}\left|A_{i}\right|+\sum_{j=1}^{m}\left|\delta_{j}\right| \ln \left|\delta_{j}\right| \geqq \sum_{i=1}^{N} \ln v_{i} .
$$

Corollary 2. If we delete assumption of 1-connectivity of $A_{1}, \ldots, A_{N}$ in Corollary 1 but retain all other assumption then

or

$$
C \sum_{i=1}^{N} d_{A_{i}}+\sum_{j=1}^{m}\left|\delta_{j}\right| \ln \left|\delta_{j}\right| \geqq \sum_{i=1}^{N} \ln v_{i}
$$

$$
C \sum_{i=1}^{N} d_{A_{i}}(\mathfrak{H})+\sum_{j=1}^{m}\left|\delta_{j}\right| \ln \left|\delta_{j}\right| \geqq \sum_{i=1}^{N} \ln v_{i} .
$$

This follows from Theorem 2, Lemma 1 and inequality (2) of 1.1.

\section{Appendix to Part 1}

\section{Calculation of Möbius Functions for Lattices}

To make this paper self-contained we give here the complete proof of the explicit formula for the Möbius function of the lattice of connected partitions of a graph.

In the general context we follow J.-C. Rota [6] correcting some inaccuracies in the proof of the main theorem. 


\section{Lattices}

Let be given finite partially-ordered set $P$ with order relation $\leqq$. We define upper bound for a set $X \subset P$ to be an element $p \in P$ such that $x \leqq p$ for all $x \in X$. Least upper bound (l.u.b.) is an upper bound which is $\leqq$ than all upper bounds.

$P$ is a lattice iff for all two of its elements $p_{1}, p_{2}$ there exists 1.u.b. $p_{1} \vee p_{2}$ and the greatest lower bound (g.l.b.) which is defined in the dual way.

Further we assume $P$ to be a lattice.

The greatest and the least element of $P$ we denote 1 and 0 respectively.

\section{Examples}

1. $P=\mathfrak{U}$ which is the set of all (non-ordered) partitions $\alpha=\left(A_{1}, \ldots, A_{k}\right)$ of the set $\{1, \ldots, n\}$. We assume that $\alpha \leqq \beta=\left(B_{1}, \ldots, B_{l}\right)$ iff each block $A_{i}$ is contained in some block $B_{j}$ of $\beta$.

2. Let $G$ be a connected graph with the set of vertices coinciding with $\{1, \ldots, n\}$ and let $\mathfrak{U}_{G} \subset \mathfrak{U}$ be the set of all partitions $\alpha=\left(A_{1}, \ldots, A_{k}\right)$ such that each block $A_{i}$ is the connected subgraph of $G$.

One can easily convince itself that $\mathfrak{A}$ and $\mathfrak{A}_{G}$ are lattices.

\section{Elementary Facts About Möbius Functions}

Let us consider the set of all (complex or real) functions $f(x, y)$ of two variables $x$, $y \in P$ such that $f(x, y)$ can be different from zero only if $x \leqq y$. This set is an algebra (incidence algebra) over (complex or real) number field with usual addition and multiplication on a scalar and with convolution-type multiplication

$$
h(x, y)=\sum_{z: x \leqq z \leqq y} f(x, z) g(z, y) .
$$
be

This algebra has an identity-Kronecker symbol $\delta(x, y)$. We define $\zeta$-function to

$$
\zeta(x, y)= \begin{cases}1, & \text { iff } x \leqq y \\ 0, & \text { otherwise }\end{cases}
$$

Lemma A.1. $\zeta$-function is invertible in the incidence algebra.

Proof. We define Möbius function $\mu_{P}=\mu(x, y)$ of the lattice $P$ by induction in the power of segment $[x, y]=\{z: x \leqq z \leqq y\}$ :

$$
\begin{aligned}
& \mu(x, x)=1, \\
& \mu(x, y)=-\sum_{z: x \leqq z<y} \mu(x, z) .
\end{aligned}
$$

One can verify that $\mu \zeta=\zeta \mu=\delta$.

Lemma A.2 (Möbius inversion formula). Let $f(x)$ be a (complex or real) function on $P$ and

$$
g(x)=\sum_{y \leqq x} f(y) .
$$


Then

$$
f(x)=\sum_{y \leqq x} g(y) \mu_{P}(y, x) .
$$

Proof.

$$
\begin{aligned}
\sum_{y \leqq x} g(y) \mu(y, x) & =\sum_{y \leqq x} \sum_{z \leqq y} f(z) \mu(y, x) \\
& =\sum_{y \leqq x} \sum_{z} f(z) \zeta(z, y) \mu(y, x)=\sum_{z} f(z) \delta(z, x)=f(x) .
\end{aligned}
$$

The following properties of Möbius functions we need in the sequel easily follow from definition (2).

1. Let $z, z^{\prime} \in[x, y]$. Then

$$
\mu_{P}\left(z, z^{\prime}\right)=\mu_{[x, y]}\left(z, z^{\prime}\right) \text {. }
$$

2. Let $P^{*}$ be partially-ordered set dual to $P$ (it is obtained by order inversion in $P$ and coincides with $P$ as a set). Then

$$
\mu_{P}(x, y)=\mu_{P *}(y, x) .
$$

3. Let $P \times Q$ be the cartesian product of lattices $P$ and $Q$, i.e. it is a set of all pairs $(p, q), p \in P, q \in Q$, and $\left(p_{1}, q_{1}\right) \leqq\left(p_{2}, q_{2}\right)$ iff $p_{1} \leqq p_{2}$ and $q_{1} \leqq q_{2}$.

Then

$$
\mu_{P \times Q}\left(\left(p_{1}, q_{1}\right),\left(p_{2}, q_{2}\right)\right)=\mu_{P}\left(p_{1}, p_{2}\right) \mu_{Q}\left(q_{1}, q_{2}\right) .
$$

4. (Inclusion-exclusion principle.) Let $P$ be the lattice of all subset $x$ of some finite set where $x \leqq y$ means $x \subset y$. Then

$$
\mu_{P}(x, y)=(-1)^{|y|-|x|}
$$

where $|x|$ is the number of elements in $x$.

This follows from the fact that $P$ is isomorphic to the product of two-point segments $[0,1]$ where $0<1$.

\section{Galois Correspondences}

Let two lattices $P$ and $Q$ be given.

We call Galois correspondence between $P$ and $Q$ a pair of mappings $\varrho: P \rightarrow Q$ and $\pi: Q \rightarrow P$ such that

1. $\varrho$ and $\pi$ invert the order;

2. $\pi(\varrho(p)) \geqq p$ and $\varrho(\pi(q)) \geqq q$ for all $p \in P, q \in Q$.

Lemma A.3. Let moreover one has

$$
\text { 1. } \pi(q)=0 \text { iff } q=1 \text {. }
$$

Then

$$
\mu_{Q}(0,1)=\sum_{a: \varrho(a)=0} \mu_{P}(0, a) .
$$


Proof. From definition of Galois correspondence it follows that $\pi(x) \geqq b$ iff $x \leqq \varrho(b)$ (e.g. if $\pi(x) \geqq b$ then $x \leqq \varrho(\pi(x) \leqq \varrho(b)$ and vice versa). Otherwise stated this means that

$$
\sum_{a \geqq b} \delta_{P}(\pi(x), a)=\zeta_{Q}(x, \varrho(b)) .
$$

We fix $x$ and consider $\zeta_{Q}(x, \varrho(b))$ and $\delta_{P}(\pi(x), a)$ as the functions on $P$ (from $b$ and $a$ respectively). We can apply then to (10) the Möbius inversion formula

$$
\begin{aligned}
\delta_{P}(\pi(x), 0) & =\sum_{a \geqq 0} \zeta_{Q}(x, \varrho(a)) \mu_{P *}(a, 0) \\
& =\sum_{a \geqq 0} \zeta_{Q}(x, \varrho(a)) \mu_{P}(0, a) .
\end{aligned}
$$

Let us put $n=\zeta-\delta$. From assumption 1 it follows that

$\delta_{P}(\pi(x), 0)=1-n_{Q}(x, 1)$.

We can rewrite (11) as

$$
1-n_{Q}(x, 1)=\zeta_{Q}(x, \varrho(0))+\sum_{a>0} \mu_{P}(0, a) \zeta_{Q}(x, \varrho(a)) .
$$

As $\varrho(0)=\varrho(\pi(1))=1$ we have $\xi_{Q}(x, \varrho(0))=1$ and then

$$
-n_{Q}(x, 1)=\sum_{a>0} \mu_{P}(0, a) \zeta_{Q}(x, \varrho(a)) .
$$

As $\mu=\delta-\mu n$ then

$$
\begin{aligned}
\mu_{Q}(0,1) & =-\sum_{0 \leqq x \leqq 1} \mu_{Q}(0, x) n_{Q}(x, 1) \\
& =\sum_{0 \leqq x \leqq 1} \sum_{a>0} \mu_{Q}(0, x) \mu_{P}(0, a) \zeta_{Q}(x, \varrho(a)) \\
& =\sum_{a>0} \mu_{P}(0, a) \delta_{Q}(0, \varrho(a))=\sum_{a: \varrho(a)=0} \mu_{P}(0, a) .
\end{aligned}
$$

Lemma A.4. Let $L$ be a lattice and a subset $R \subset L$ be given such that: $0 \bar{\epsilon} R$ and for each $x \bar{E} L, x \neq 0$ there exists $y \in R$ such that $y \leqq x$. Let $k \geqq 2$ and $q_{k}-$ the number of subsets $X \subset R$ such that $|X|=k$ and l.u.b. of $X$ is equal to 1. Then

$$
\mu_{L}(0,1)=q_{2}-q_{3}+q_{4}-\ldots
$$

Proof. In the Lemma A.3 we take $Q=L^{*}$ and $P$ - the lattice of all subsets of $R$.

Also we take $\pi(x), x \in Q$, as the subset of all elements of $R$ which $\leqq x$ in $L$. In particular $\pi(1)=0$ (empty set).

For $A \in R$ we take $\varrho(A)$ equal to the 1.u.b. of $A$ in $L$ (i.e. g.l.b. of $A$ in $Q=L^{*}$ ). Then (12) follows from Lemma A.3 and from (8).

\section{Explicit Calculation of Möbius Function}

We define the rank $r(p)$ of element $p \in P$ as maximal number $r$ minus 1 such that there exists a sequence $x_{1}, \ldots, x_{r} \in P$ such that $0=x_{1}<x_{2}<\ldots<x_{r}=p$. We call atoms the elements of rank 1. 
Let $R$ be the set all atoms of $P$. We call $P$ a geometric lattice (equivalently, $M$-lattice) iff:

1. arbitrary element of $P$ is the l.u.b. of some set of atoms (except 0 );

2. if $p$ is an atom and $a$ is arbitrary then either $p \leqq a$ or $p \vee a$ covers $a$ (i.e. there is no $x$ such that $a<x<p \vee a$ ).

Other equivalent definitions see in [11].

Let us call a set $X$ of atoms independent iff $r$ (l.u.b. of $X)=|X|$ and call it generating iff l.u.b. of $X$ is 1 .

In the geometric lattice with $r(1)=n-1$ any independent generating subset of atoms contains exactly $n-1$ elements.

Remark A.1. It is not difficult to prove that $\mathfrak{U}$ and $\mathfrak{U}_{G}$ are geometric lattices. The last property for them is easy to prove directly (so we shall not prove it in the general case). If between any two vertices of graph $G$ there is not more than one line (edge) then the set of atoms of $\mathfrak{A}_{G}$ can be identified with lines of $G: \alpha$ is an atom iff all its blocks contain one element except one which contains two; we identify $\alpha$ with a line which connects these two elements.

Let us call a circuit $T$ a subset of atoms such that $T$ is not independent but all its (proper) subsets are independent.

Remark A.2. $T$ is a circuit in $\mathfrak{A}_{G}$ iff the corresponding set of lines of $G$ (constructed in Remark A.1) is the closed path without selfintersections, i.e. each vertex is incident either with 2 or 0 lines of $T$.

Proof. Such a path $C$ is not independent that is why if $T \supset C$ then $T=C$.

We assume now that the set of all atoms of $P$ is enumerated is some fixed way: $a_{1}, \ldots, a_{k}$.

If $T=\left\{a_{i_{1}}, \ldots, a_{i_{e}}\right\}$ is a circuit and $i_{1}<\ldots<i_{e}$ then we shall call $T^{\prime}=\varphi(T)$ $=\left\{a_{i_{1}}, \ldots, a_{i_{e-1}}\right\}$ a broken circuit.

Let us denote $\varphi^{-1}\left(T^{\prime}\right)$ some circuit $T$ such that $\varphi(T)=T^{\prime}$. In the situation of Remark A.2 one can see that such current is unique.

Theorem A.5 (J.-C. Rota). Let $P$ be a geometric lattice. Then

$$
\mu_{P}(0,1)=(-1)^{r(1)} m_{1}
$$

where $m_{1}$ is the number of subsets $X \subset R$ of atoms such that

1. $|X|=r(1)$;

2. $X$ does not contain any broken circuit.

Proof $^{1}$. For the fixed enumeration of the set $R$ of atoms we can order the set of all broken circuits $T_{1}^{\prime}, \ldots, T_{\sigma}^{\prime}$ in such a way that (and otherwise arbitrary)

$$
f\left(T_{i}^{\prime}\right) \leqq f\left(T_{j}^{\prime}\right) \quad \text { if } \quad i<j
$$

where $f\left(T^{\prime}\right)=i_{k}$ is the number (in fixed enumeration of $R$ ) of the last atom in $T^{\prime}=\left\{a_{i_{1}}, \ldots, a_{i_{k}}\right\}, i_{1}<\ldots<i_{k^{\prime}}$.

1 The presentation of this proof in [6] is wrong but the ideas can be restored. This is done here 
Let us denote $S_{i}$ - the set of all generating subsets $X \subset R$ such that $X$ contains $T_{i}$ but does not contain neither of $T_{1}^{\prime}, T_{2}^{\prime}, \ldots, T_{i-1}^{\prime}$;

$R_{j}^{i}$, the set of all generating subsets $X \subset R$ such that $|X|=j$ and $X$ does not contain $T_{1}^{\prime}, T_{2}^{\prime}, \ldots, T_{i}^{\prime}$.

$R_{j}^{i, A}$, the set of all generating subsets $X \subset R$ such that $|X|=j, X$ does not contain $T_{1}^{\prime}, \ldots, T_{i-1}^{\prime}$ but contains circuit $\varphi^{-1}\left(T_{i}^{\prime}\right)$.

$R_{j}^{i, B}$, the set of all generating subsets $X \subset R$ such that $|X|=j, X$ does not contain $T_{1}^{\prime}, \ldots, T_{i-1}^{\prime}$, contains $T_{i}^{\prime}$ but does not contain circuit $\varphi^{-1}\left(T_{i}^{\prime}\right)$.

Then for $i=0,1, \ldots$ one has

$$
\left|R_{j}^{i}\right|=\left|R_{j}^{i+1}\right|+\left|R_{j}^{i+1, A}\right|+\left|R_{j}^{i+1, B}\right| \text {. }
$$

Let us prove that for all $i=0,1, \ldots$

$$
\mu_{P}(0,1)=\left|R_{2}^{i}\right|-\left|R_{3}^{i}\right|+\left|R_{4}^{i}\right|-\ldots
$$

by induction in $i$. For $i=0$ this is Lemma A.4. For any $i>0$ we have by induction and due to formula (14)

$$
\begin{aligned}
\mu(0,1)= & \left|R_{2}^{i-1}\right|-\left|R_{3}^{i-1}\right|+\left|R_{4}^{i-1}\right|-\ldots \\
= & \left|R_{2}^{i}\right|-\left|R_{3}^{i}\right|+\left|R_{4}^{i}\right|-\ldots+\left|R_{2}^{i, A}\right| \\
& +\left(\left|R_{2}^{i, B}\right|-\left|R_{3}^{i, A}\right|\right)-\left(\left|R_{3}^{i, B}\right|-\left|R_{4}^{i, A}\right|\right)+\ldots .
\end{aligned}
$$

But $\left|R_{2}^{i, A}\right|=0$ as circuit cannot contain exactly two elements (any two atoms form independent subset).

We define one-to-one correspondence $\zeta$ between $R_{j}^{i, B}$ and $R_{j+1}^{i, A}$ in the following way: for $X \in R_{j}^{i, B}$ we put

$$
\zeta(X)=X \cup\left(\varphi^{-1}\left(T_{i}^{\prime}\right)-T_{i}^{\prime}\right) .
$$

$\zeta(X) \in R_{j+1}^{i, A} \quad$ as element $a_{e}=\varphi^{-1}\left(T_{i}^{\prime}\right)-T_{i}^{\prime} \quad$ cannot belong to any $T_{1}^{\prime}, \ldots, T_{i-1}^{\prime}$ due to chosen enumeration of the system of broken circuits.

For if $j<i$ and $a_{k} \in T_{j}^{\prime}$ then

$$
l>f\left(T_{i}^{\prime}\right) \geqq f\left(T_{j}^{\prime}\right) \geqq k \text {. }
$$

From (15) it follows for $i=\sigma$ that

$$
\left.\mu_{P}(0,1)=(-1)^{r(1)} \mid R_{r(1)}^{\sigma}\right\}
$$

as if $X$ does not contain any broken circuit (and so no circuit) then $X$ is independent. As $X$ is generating at the same time then it contains exactly $r(1)$ elements. So $\left|R_{j}^{\sigma}\right|=0$ if $j \neq n-1$.

The theorem is proved.

Corollary A.1. In the situation of Remark A.2 one has

$$
\mu_{\mathfrak{A}_{G}}(0,1)=(-1)^{n-1} m_{1}
$$

where $m_{1}$ is the number of subsets $X$ of lines of $G$ such that $|X|=n-1$ and $X$ does not contain any broken circuit. 


\section{Part 2}

\subsection{The Main Result}

Let us consider probability measure $\mu$ on $S^{\mathbb{Z}^{v}}$ where $S$ is a set of spin values (a measurable space) for each point of $\mathbb{Z}^{v} ;\langle\cdot\rangle$-expectation with respect to $\mu$.

Definition 1. Measure $\mu$ is said to have cluster expansion with respect to given $\mathfrak{U}$ if for each local $\psi_{B}$ there is an expansion

$$
\left\langle\psi_{B}\right\rangle=\sum_{R} b_{R}, \quad b_{R}=b_{R}\left(\psi_{B}\right)
$$

where the summation is through all $R$ such that $R$ is a union of finite number of $\tilde{\Gamma}_{i}$ where $\Gamma_{i}$ is a connected array with elements in $\mathfrak{U}$ and such that $\tilde{\Gamma}_{i} \cap B \neq \emptyset$. It is required that for sufficiently small $\lambda>0$

$$
\left|b_{R}\left(\psi_{B}\right)\right| \leqq C\left(\psi_{B}\right)(C \lambda)^{\delta_{R}^{(B)}(\mathfrak{R})}
$$

from which follows absolute convergence of (1) if $C\left(\psi_{B}\right)<\infty$.

Let now local $\psi_{B_{1}}, \ldots, \psi_{B_{n}}$ be given. A cross section $\zeta$ is a function which to each $D \subset\{1, \ldots, n\}$ associates some $R=\zeta(D) \subset \mathbb{Z}^{v}$ such that :

1. $b_{\zeta(\{i\})}\left(\psi_{B_{i}}\right) \neq 0$ for all $i=1, \ldots, n$;

2. $\zeta(P)=\bigcup_{i \in D} \zeta(\{i\})$.

We shall define $G_{\zeta}$ with vertices $\{1, \ldots, n\}$ where vertices $i$ and $j$ are connected with a line iff

$$
\left[B_{i} \cup \zeta(\{i\})\right] \cap\left[B_{j} \cup \zeta(\{j\})\right] \neq \emptyset .
$$

Given $\psi_{B_{1}}, \ldots, \psi_{B_{n}}$ and cross-section $\zeta$ we define $f_{\zeta}(D)$ to be virtual field on $\{1, \ldots, n\}$ :

$$
f_{\zeta}(D)=\frac{1}{m_{\zeta}(D)} b_{\zeta(D)}\left(\sum_{i \in D} \psi_{B_{\imath}}\right)
$$

where $m_{\zeta}(D)$ is the number of different restrictions $\zeta_{D}^{\prime}$ onto $D$ of all possible crosssections ' $\zeta^{\prime}$ such that for this $D$

$$
\zeta(D)=\bigcup_{i \in D} \zeta^{\prime}(\{i\})
$$

Theorem 1. Assume that $\mu$ has a cluster expansion and each cross-section defines independent virtual field $f_{\zeta}(D)$ with respect to $G_{\zeta}$. Then for $\lambda$ sufficiently small the following uniform cluster estimate take place

$$
\left|\left\langle\psi_{B_{1}}, \ldots, \psi_{B_{n}}\right\rangle\right| \leqq C_{\psi} C^{\sum_{i=1}^{n} d_{B_{i}}(\mathfrak{2})}(C \lambda)^{d_{21}\left(B_{1}, \ldots, B_{n}\right)} \prod_{i=1}^{n} u_{i}
$$

where $C_{\psi} \equiv C_{f}(\{1, \ldots, n\})$ is defined in (8) 1.2 for

$$
f(D)=\left\langle\prod_{i \in D} \psi_{B_{i}}\right\rangle
$$

$d_{\mathfrak{A}}\left(B_{1}, \ldots, B_{n}\right)$ is the least such $d$ that there exist $A_{1}, \ldots, A_{d} \in \mathfrak{U}$ such that collection $\Gamma=\left(B_{1}, \ldots, B_{n}, A_{1}, \ldots, A_{d}\right)$ is connected. 
Proof. Given momentae of virtual field $f_{\zeta}(D)$ one can define semiinvariants $g_{\zeta}(D)$ using (1) or (4) of 1.2.

\section{Lemma 1.}

$$
\left\langle\psi_{B_{1}}, \ldots, \psi_{B_{n}}\right\rangle=\sum_{\zeta} g_{\zeta}(\{1, \ldots, n\}) .
$$

We have by definition

$$
\left\langle\psi_{B_{1}} \ldots, \psi_{B_{n}}\right\rangle=\sum f\left(D_{1}\right) \ldots f\left(D_{k}\right)(-1)^{k-1}(k-1) !
$$

and

$$
\begin{aligned}
\left\langle\psi_{B_{1}}, \ldots, \psi_{B_{n}}\right\rangle_{\zeta} & \stackrel{\text { def }}{=} g_{\zeta}(\{1, \ldots, n\}) \\
& =\sum f_{\zeta}\left(D_{1}\right) \ldots f_{\zeta}\left(D_{k}\right)(-1)^{k-1}(k-1) !
\end{aligned}
$$

where in (9) and (10) the summation is through all partitions $\left(D_{1}, \ldots, D_{k}\right)$ of the set $\{1, \ldots, n\}$.

To prove (8) it is sufficient to prove

$$
f\left(D_{1}\right) \ldots f\left(D_{k}\right)=\sum_{\zeta} f_{\zeta}\left(D_{1}\right) \ldots f_{\zeta}\left(D_{k}\right)
$$

for any fixed partition $\left(D_{1}, \ldots, D_{k}\right)$.

Let us insert into the left hand side of (11) expansion (1) for $f(D)$.

Then we obtain from the left the sum of terms

$$
b_{R_{1}}\left(\prod_{i \in D_{1}} \psi_{B_{i}}\right) \ldots b_{R_{k}}\left(\prod_{i \in D_{k}}\left(\psi_{B_{i}}\right)\right) \text {. }
$$

In the right hand side of (11) let us consider only those $\zeta$ for which

$$
R_{i}=\zeta\left(D_{i}\right) \text { for } i=1, \ldots, k \text {. }
$$

There is exactly $m_{\zeta}\left(R_{1}\right) \ldots m_{\zeta}\left(R_{k}\right)$ of such $\zeta$. Lemma 1 follows from this if we insert expressions (4) into right hand side of (11).

Let us use now independence of virtual field $f_{\zeta}(D)$. Then

$$
g_{\zeta}(D)=0
$$

if subgraph $D_{\zeta}$ of $G_{\zeta}$ with vertices in $D$ is not connected. That is why one can use Möbius inversion formula as in 1.2 for lattice $\mathfrak{U}_{G_{\zeta}}$ (its Möbius function is denoted $\left.\mu_{\zeta}\right)$ :

$$
\left\langle\psi_{B_{1}}, \ldots, \psi_{B_{n}}\right\rangle_{\zeta}=\sum_{\delta} \mu_{\zeta}(\delta, 1) f_{\zeta}\left(D_{1}, \ldots, D_{k}\right)
$$

where the summation is through all partitions $\delta=\left(D_{1}, \ldots, D_{k}\right) \in \mathfrak{A}_{G \zeta^{*}}$.

From here using (8), (13), Lemma 2 of 1.2 and formula (14) of 1.2 we get

$$
\left|\left\langle\psi_{B_{1}}, \ldots, \psi_{B_{n}}\right\rangle\right| \leqq \sum_{\zeta}^{\prime} \sum_{\delta=\left(D_{1}, \ldots, D_{k} \in \mathfrak{A}_{G_{\xi}}\right.} \prod_{i=1}^{k} v_{i}(\zeta, \delta) f_{\zeta}\left(D_{i}\right)
$$

where in $\sum^{\prime}$ the summation is through all $\zeta$ such that $G_{\zeta}$ is connected; $v_{i}(\zeta, \delta)$ - the number of $j=1, \ldots, k$ such that

$$
\left[\zeta\left(D_{i}\right) \cup\left(\bigcup_{l \in D_{l}} B_{l}\right)\right] \cap\left[\zeta\left(D_{j}\right) \cup\left(\bigcup_{l \in D_{J}} B_{l}\right)\right] \neq \emptyset
$$


The right-hand side of (15) can be bounded by

$$
\sum_{\alpha} \sum_{R_{1}, \ldots, R_{k}} \prod_{i=1}^{k}\left|b_{R_{l}}\left(\prod_{l \in \alpha_{i}} \psi_{B_{l}}\right)\right| \mid v_{i}\left(R_{1} \ldots, R_{k}\right)
$$

where $v_{i}\left(R_{1}, \ldots, R_{k}\right)$ is the number of $j=1, \ldots, k$ such that

$$
\left(R_{i} \cup N_{i}\right) \cap\left(R_{j} \cap N_{j}\right) \neq \emptyset, \quad N_{i}=\bigcup_{l \in \alpha_{l}} B_{l} .
$$

The summation in (16) is through all partitions $\alpha=\left(\alpha_{1}, \ldots, \alpha_{k}\right)$ of $\{1, \ldots, n\}$ and all $R_{1}, \ldots, R_{k} \subset \mathbb{Z}^{v}$ such that:

1. The following graph $G\left(R_{1}, \ldots, R_{k}\right)$ is connected: it has vertices $\{1, \ldots, k\}$ and a line between $i$ and $j$ iff

$$
\left(R_{i} \cup N_{i}\right) \cap\left(R_{j} \cup N_{j}\right) \neq \emptyset ;
$$

2. for some $\zeta$

$R_{i}=\zeta\left(D_{i}\right), \quad i=1, \ldots, k$.

We can delete condition 2 and leave only more weak condition.

$2^{\prime}$. For all $i=1, \ldots, k$ there exists a collection $\Gamma_{i}$ of sets belonging to $\mathfrak{A}$ such that a collection $\left(\Gamma_{i}, B_{l}: l \in \alpha_{i}\right)$ is connected and $\tilde{\Gamma}_{i}=R_{i}$.

We shall bound (16) under conditions 1 and $2^{\prime}$.

Using (1) we have

$$
\prod_{i=1}^{k}\left|b_{R_{i}}\left(\prod_{l \in \alpha_{i}} \psi_{B_{l}}\right)\right| \leqq C_{\psi}(C \lambda)^{\sum_{i=1}^{k} \delta_{R_{i}}^{\alpha_{i}(\mathfrak{I})}} \text {. }
$$

For "all $\alpha$ and $i=1, \ldots, k$ we choose a point $t_{i}(\alpha)$ which is the first in lexicographic order among points of $\bigcup_{l \in \alpha_{l}} B_{l}$. We denote a collection of these points
for a given $\alpha$

$$
\eta(\alpha)=\left(t_{1}(\alpha), \ldots, t_{k}(\alpha)\right) \text {. }
$$

Let us put for any collection $\eta$ of points

$$
u(\eta)=\sum_{t \in \eta} \ln u_{t}
$$

where $u_{t}$ is the number of $t^{\prime} \in \eta$ such that $t=t^{\prime}$.

Using Corollary 2 of 1.3 we get

$$
\prod_{i=1}^{k} v_{i}\left(R_{1}, \ldots, R_{k}\right) \leqq \exp [u(\eta(\alpha))] C^{\sum_{=1}^{k} d_{Q_{i}}(\mathfrak{R})}
$$

where

$$
Q_{i}=R_{i} \cup N_{i},
$$

In view of condition $2^{\prime}$

$$
\begin{aligned}
d_{Q_{i}}(\mathfrak{U}) \leqq & \delta_{R_{i}}^{\alpha_{i}}(\mathfrak{U})+d_{N_{l}}(\mathfrak{U}) \leqq \delta_{R_{l}}^{\alpha_{i}}(\mathfrak{U}) \\
& +\sum_{l \in \alpha_{l}} d_{B_{l}}(\mathfrak{U})+d_{\mathfrak{A}}\left(B_{l}: l \in \alpha_{i}\right)
\end{aligned}
$$

where $d_{\mathfrak{Q}}\left(B_{l_{1}}, B_{l_{2}}, \ldots\right)$ is defined in Theorem 1 . 
Then

$$
\sum_{i=1}^{k} d_{Q_{i}}(\mathfrak{U}) \leqq \sum_{i=1}^{k} \delta_{R_{\imath}}^{\alpha_{\imath}}(\mathfrak{A})+\sum_{j=1}^{n} d_{B_{J}}(\mathfrak{A})+d_{\mathfrak{U}}\left(B_{1}, \ldots, B_{n}\right) .
$$

Using (17), (18), (20) one can bound (16) by

$$
\begin{gathered}
C_{\psi} \exp \left[\sum_{i=1}^{n} \mathrm{~d}_{B_{i}}(\mathfrak{A}) \ln C+d_{\mathfrak{Q}}\left(B_{1}, \ldots, B_{n}\right) \ln C\right] \sum_{\alpha} \exp [u(\eta(\alpha))] \\
\cdot \sum_{R_{1}, \ldots, R_{k}} \exp \left[\sum_{i=1}^{k} \delta_{R_{\imath}}^{\alpha_{i}}(\mathfrak{U}) \ln (\lambda C)\right] .
\end{gathered}
$$

One can perform now summation through all $R_{1}, \ldots, R_{k}$ satisfying conditions $1.2^{\prime}$ for fixed $\alpha$. It gives

$$
\begin{aligned}
& \sum_{R_{1}, \ldots, R_{k}}(C \lambda)^{\sum_{\equiv}^{k} \delta_{1}^{\alpha_{i}}(\mathfrak{I})} \\
& \leqq C_{1}^{\sum_{i}^{k}\left|B_{1}\right|}\left(C_{2} \lambda\right)^{d_{2 l}\left(B_{1}, \ldots, B_{n}\right)}
\end{aligned}
$$

with $C_{1}$ and $C_{2}$ not depending on $\alpha$.

This follows from Lemma 4 of 1.1.

Then (21) is bounded by

$$
\begin{gathered}
C_{\psi} \exp \left[\sum_{i=1}^{n} d_{B_{i}}(\mathfrak{U}) \ln C+d_{\mathfrak{N}}\left(B_{1}, \ldots, B_{n}\right) \ln (C \lambda)\right] \\
\cdot \sum_{\alpha} \exp [u(\eta(\alpha))] .
\end{gathered}
$$

We shall bound the last sum. Let $\beta_{i}, i=1, \ldots, m$, be groups of equal points among $t_{1}, \ldots, t_{n}, \beta_{1}+\ldots+\beta_{m}=n$ where $t_{i}$ is first point of $B_{i}$ in lexicographic order. It is easy to see that

$$
\sum_{\alpha} \exp [u(\eta(\alpha))] \leqq \prod_{i=1}^{m} \sum_{\delta_{\mathrm{t}}}\left(\left|\delta_{i}\right|^{\left|\delta_{i}\right|}+1\right)
$$

where $\delta_{i}$ runs all partitions of $\beta_{i}$. Right-hand side of (24) can be bounded by

$$
C^{n} \prod_{i=1}^{m}\left|\beta_{i}\right|^{\left|\beta_{i}\right|}
$$

what follows e.g. from Theorem 1 of 1.2 for the case of complete (with all possible lines) graph $G$ but can be easily proved directly. Inserting (25) into (23) we get due to

$$
\exp \sum\left|\beta_{i}\right| \ln \left|\beta_{i}\right| \leqq \prod_{i=1}^{n} v_{i}
$$

and to Corollary 2 of 1.3 the result of Theorem 1 . 


\subsection{High-Temperature Expansions}

We prove here that conditions of Theorem 1, 2.1 are fullfilled for a large class of lattice spin systems in hight-temperature region.

Let for each point $t \in \mathbb{Z}^{v}$ some set $S_{t}$ of "spin values" be given. We may assume $S_{t}$ to be equal to $S$. Let $\Sigma_{t}$ be some $\sigma$-algebra of subsets of $S_{t}$ and $\mu_{t}^{0}$-probability measure on $\left(S_{t}, \Sigma_{t}\right)$. Let us put

$$
\Omega=\underset{t \in \mathbb{Z}^{v}}{\times} S_{t}, \quad \Sigma_{A}=\underset{t \in A}{\times} \Sigma_{t}, \quad \Sigma_{\mathbb{Z}^{v}}=\Sigma, \quad \mu^{0}=\underset{t}{\times} \mu_{t}^{0},
$$

$\langle\cdot\rangle_{0}$-expectation with respect to $\mu^{0}$. A function $F$ on $\Omega$ is called local if it is measurable w.r.t. $\Sigma_{A}$ for some finite $A$. We write $F \equiv F_{A}$ in this case.

Let a system $\mathfrak{U}$ of finite subsets of $\mathbb{Z}^{v}$ with uniformly bounded diameter be given and for each $A \in \mathfrak{U}$ is given a local function $\Phi_{A}$. A collection $\Phi=\left(\Phi_{A}\right)$ is called a potential.

Let us put

$$
k_{A}=\exp \left(-\lambda \Phi_{A}\right)-1, \quad k_{\Gamma}=\prod_{A \in \Gamma} k_{A}
$$

for each non-ordered array $\Gamma=\left(A_{1}, \ldots, A_{k}\right)$ with $A_{i} \in \mathfrak{U}$ (we suppose here that $A_{i}$ are mutually different).

Here constants $C, C_{i}, \lambda_{0}$ depend only on the situation in question, i.e. on $v, \mathfrak{A}$, $\Phi$.

We assume in this paragraph that there exist constants $\lambda_{0}>0$ and $C>0$ such that uniformly for $0<\lambda<\lambda_{0}$ and for all $\Gamma$

$$
\left|\left\langle k_{\Gamma}\right\rangle_{0}\right| \leqq(C \lambda)^{|\Gamma|}
$$

We specify a class of potentials for which (1) is fullfilled and which is sufficient for most applications.

Functions $\Phi_{A}$ in question are uniformly bounded from below and have a representatiọn

$$
\Phi_{A}=\sum_{\alpha=1}^{N_{A}} \varphi_{\alpha, t_{1}}^{(A)} \ldots \varphi_{\alpha, t_{n}}^{(A)}
$$

where $A=\left\{t_{1}, \ldots, t_{n}\right\}, N_{A}$ and expectations $\left\langle\left|\varphi_{\alpha, t_{2}}^{(A)}\right|^{p}\right\rangle_{0}$ for suitable fixed $p$ are bounded uniformly in $\alpha, A, t_{i}$.

With the inequality

$$
\begin{aligned}
\left\langle k_{\Gamma}\right\rangle_{0} \mid & =\left|\left\langle\prod_{A \in \Gamma} \int_{0}^{\lambda} e^{-\lambda^{\prime} \Phi_{A}}\left(-\Phi_{A}\right) d \lambda^{\prime}\right\rangle_{0}\right| \\
& \leqq\left(C_{1} \lambda\right)^{|\Gamma|}\left\langle\prod_{A \in \Gamma}\left|\Phi_{A}\right|\right\rangle_{0}
\end{aligned}
$$

one gets (1) for this class of potentials.

We remind that Gibbs measure $\mu_{V}$ is given by the density

$$
\begin{gathered}
\frac{d \mu_{V}}{d \mu^{0}}=Z_{V}^{-1} \exp \left(-\lambda U_{V}\right), \\
U_{V}=\sum_{A \in \mathfrak{A}, A \subset V} \Phi_{A},\langle\cdot\rangle_{V} \text {-expectation w.r.t. } \mu_{V} .
\end{gathered}
$$


If for each local bounded $F$

$\lim _{V}\langle F\rangle_{V}=\langle F\rangle$

exists it defines limit Gibbs measure $\mu\left(V \rightarrow \mathbb{Z}^{v}\right.$ in the sense of van Hove).

Theorem 1. Under the condition (1) measure $\mu$ has cluster expansion and conditions of Theorem 1 in 2.1 are fullfilled for $0 \leqq \lambda<\lambda_{0}$ with $\lambda_{0}>0$ sufficiently small.

To prove this theorem we shall get first equations of Kirkwood-Salsburg type for what we use the following formula. We fix $B \subset V$

$$
\begin{aligned}
\exp \left(-\lambda U_{V}\right) & =\prod_{A \subset V}\left(1+k_{A}\right) \\
& =\sum_{\Gamma} k_{\Gamma}=\sum_{\Gamma}^{\prime} k_{\Gamma} \exp \left(-\lambda U_{V-(B \cup \tilde{I})}\right)
\end{aligned}
$$

where in the first sum the summation is through all $\Gamma$ (including empty one) and in $\sum^{\prime}$ is through all $\Gamma=\left(A_{1}, \ldots, A_{k}\right)$ such that collection $(B, \Gamma)=\left(B, A_{1}, \ldots, A_{k}\right)$ is connected.

We consider now the class of all local $\psi_{B}$ with finite momentae.

Inserting (4) into $\left\langle\psi_{B} \exp \left(-\lambda U_{V}\right)\right\rangle_{0}$ dividing on $Z_{V}$ and passing to the limit we get

$$
\begin{aligned}
\left\langle\psi_{B}\right\rangle & =\sum_{\Gamma}^{\prime}\left\langle\psi_{B} k_{\Gamma}\right\rangle_{0} f_{B \cup \tilde{\Gamma}}, \\
f_{B \cup \tilde{\Gamma}} & =\lim _{V} f_{B \cup \tilde{\Gamma}}^{(V)}, \\
f_{B}^{(V)} & =Z_{V-B} / Z_{V}, \quad B \subset V .
\end{aligned}
$$

Let us fix now for each nonempty finite $A \subset \mathbb{Z}^{v}$ some point $t_{A} \in A$. Then we get

$$
\begin{aligned}
& f_{A}=f_{A-t_{A}}-\sum_{\Gamma}^{\prime \prime}\left\langle k_{\Gamma}\right\rangle_{0} f_{A \cup \tilde{\Gamma}}, \\
& f_{\emptyset}=1
\end{aligned}
$$

using (4) for $f_{A-t}$. In $\sum^{\prime \prime}$ the summation is through all connected $\Gamma$ such that $t_{A} \in \tilde{\Gamma}$ and $\tilde{\Gamma} \subset \mathbb{Z}^{v}-\left(A-t_{A}\right)$.

In [7] we used the ideas of [8] to prove uniqueness of solution of (6) and other properties. All these results will easily follow from considerations below and we do not stay on it.

We derive the explicit series for $\left\langle\psi_{B}\right\rangle$ and $f_{A}$ here.

We first specify a correspondence $A \rightarrow t_{A}$.

Let $\mathscr{B}$ be the collection of all finite subsets of $\mathbb{Z}^{v}$. We force $\mathscr{B}$ to be graph such that there is a line between $B_{1}, B_{2} \in \mathscr{B}$ iff $B_{1}=B_{2} \backslash t_{B_{1}}$ or vice versa. We note that $\mathscr{B}$ is a connected tree. Vertex $\emptyset$ is the lowest vertex of $\mathscr{B}$. For each vertex $B \in \mathscr{B}$ there is exactly $|B|$ vertices below $B$.

We require that

1. For each $B \in \mathscr{B}$ a point $t_{B}$ lies on the boundary of $B$ [i.e. euclidean distance $\left.\varrho\left(t_{B}, \mathbb{Z}^{v} \backslash B\right)=1\right]$.

2. If $B_{1} \cup \ldots \cup B_{n}$ is a partition of $B$ onto its 1 -connected components then $t_{B}=t_{B_{l}}$ for some $i$. 
It is easy to achieve the performance of this conditions by induction in $|B|$. Let us consider finite sequences of pairs

$$
\gamma=\left[\left(B_{1}, \Gamma_{1}\right), \ldots,\left(B_{q}, \Gamma_{q}\right)\right], \quad q=0,1, \ldots,
$$

where

$$
B_{i}=B_{i}(\gamma) \in \mathscr{B}, \quad \Gamma_{i}=\Gamma_{i}(\gamma)
$$

are collections of pairwise different sets $A \in \mathfrak{A}$ and moreover:

1. For $p>1 B_{p}$ lies below $B_{p-1} \cup \tilde{\Gamma}_{p-1}$ in $\mathscr{B}$.

2. For $p>1 \Gamma_{p}$ is a nonempty connected collection such that

$$
t_{B_{p}} \in \tilde{\Gamma}_{p} \subset \mathbb{Z}^{v}-\left(B_{p}-t_{B_{p}}\right) \text {. }
$$

\section{Lemma 1.}

$$
\left\langle\psi_{B}\right\rangle=\sum_{\gamma} a_{\gamma}
$$

where

$$
a_{\gamma}=\left\langle\psi_{B} k_{\Gamma_{1}(\gamma)}\right\rangle_{0}\left\langle-k_{\Gamma_{2}(\gamma)}\right\rangle_{0} \ldots\left\langle-k_{\Gamma_{q}(\gamma)}\right\rangle_{0}
$$

and the summation is through all $\gamma$ satisfying conditions 1,2 and the following condition 3. $B_{1}(\gamma)=B$ and $\Gamma_{1}(\gamma)$ may be empty or such that collection $\left(B, \Gamma_{1}\right)$ is connected.

Moreover series $\sum\left|a_{\gamma}\right|$ is convergent.

Proof. Let us consider system (6).

Iterating it we get its explicit solution

$$
f_{A}=1+\sum_{\gamma} \tilde{a}_{\gamma}
$$

where

$$
\tilde{a}_{\gamma}=\left\langle-k_{\Gamma_{1}}\right\rangle_{0} \ldots\left\langle-k_{\Gamma_{q}}\right\rangle_{0}
$$

satisfy condition 1 and condition 2 but for $q \geqq 1$ and moreover $B_{1} \subset A$. (7) follows from (8) and (5).

For proof of absolute convergence we use (1). Then

$$
\left|a_{\gamma}\right| \leqq C\left(\psi_{B}\right)(C \lambda)^{\left|\Gamma_{1}\right|+\ldots+\left|\Gamma_{q}\right|}
$$

where, e.g. if $\psi_{B}$ is bounded, then

$$
C\left(\psi_{B}\right)=\sup \left|\psi_{B}\right|
$$

or otherwise in $L_{2}\left(d \mu^{(0)}\right)$

$$
C\left(\psi_{B}\right)=\left\|\psi_{B}\right\|_{2}^{(0)} \text {. }
$$

We note that uniformly in $\psi_{B}$ with finite $\left\langle\left|\psi_{B}\right|^{2}\right\rangle_{0}$

$$
\mid\left\langle\psi_{B} k_{\Gamma}\right\rangle_{0} \leqq C\left(\psi_{B}\right)(C \lambda)^{|\Gamma|}
$$

We need also 
Lemma 2. The number of $\Gamma$ such that $(B, \Gamma)$ is connected and $|\Gamma|=N$ does not exceed $2^{|B|} C^{N}$.

Proof. Let $\Gamma_{1}, \ldots, \Gamma_{r}$ be maximal connected subcollections of $\Gamma$ (and thus uniquely determined). For each $i=1, \ldots, r$ we choose the first in lexicographic order point $t_{i} \in \tilde{\Gamma}_{i}$ among points of $\tilde{\Gamma}_{i} \cap B$.

The number of arrays $\left(t_{1}, \ldots, t_{r}\right)$ does not exceed $2^{|B|}$. The number of different $\Gamma_{i}$ for given $t_{i}$ is bounded in Lemma 2, 1.1 .

We return to the proof of Lemma 1 and denote

$$
r_{i}=\left|B_{i} \cup \tilde{\Gamma}_{i}-B_{i+1}\right|=\left|B_{i}\right|+\left|\tilde{\Gamma}_{i}\right|-1-\left|B_{i+1}\right|,
$$

i.e. $r_{i}$ defines the degree of descent along the tree $\mathscr{B}$ in choosing $B_{i+1}$.

We have

$$
\sum_{i=1}^{q-1} r_{i} \leqq|B|+\sum_{i}\left|\tilde{\Gamma_{i}}\right|
$$

From this follows that the number of different sequences $\left(r_{1}, \ldots, r_{q-1}\right)$ does not exceed $2^{|B|+\left|\tilde{\Gamma}_{1}\right|+\ldots+\left|\tilde{\Gamma}_{q}\right|}$.

Collecting all estimates we derive that the number of sequences $\gamma$ such that

$$
\left|\Gamma_{1}(\gamma)\right|=N_{1}, \ldots,\left|\Gamma_{q}(\gamma)\right|=N_{q}
$$

does not exceed

$$
4^{|B|} C^{N_{1}+\ldots+N_{q}} \text {. }
$$

From (9) and (10) absolute convergence of series (7) evidently follows. Lemma 1 is proved.

The support of $\gamma$ is defined to be

$$
\operatorname{supp} \gamma=\tilde{\Gamma}_{1} \cup \ldots \cup \tilde{\Gamma}_{q} \text {. }
$$

We resumm now series (7) denoting

$$
\begin{aligned}
& b_{R}\left(\psi_{B}\right) \equiv b_{R}=\sum_{\gamma: \operatorname{supp} \gamma=R} a_{\gamma}, \\
& b_{R}\left(\psi_{B}\right)=0 \text { if }\{\gamma: \operatorname{supp} \gamma=R\} \text { is empty, } \\
& \left(b_{\emptyset}=a_{\emptyset}=\left\langle\psi_{B}\right\rangle_{0}\right) .
\end{aligned}
$$

The resummed series is

$$
\left\langle\psi_{B}\right\rangle=\sum_{R} b_{R} .
$$

This resummed series gives desired cluster expansion. It is not difficult to persuade oneself that the independence of virtual fields-cross sections is also fullfilled.

\subsection{Contour Cluster Expansions for Sufficiently Low Temperatures}

We consider here only Ising model with interaction

$$
U=-\beta \sum_{\left|t-t^{\prime}\right|=1} \sigma_{t} \sigma_{t^{\prime}}, \quad \sigma_{t}= \pm 1
$$


for $\beta>0$ sufficiently large but the same methods are applicable to the other contour models. We suppose the definition of contours for this model to be known and simplest properties of this model also. Let us consider this system in volume $V$ with $(+)$-boundary conditions or in infinite volume which is the limit of these systems with $(+)$-boundary conditions.

We shall consider non-ordered collections of contours $\vartheta=\left(\Gamma_{1}, \ldots, \Gamma_{s}\right)$ which can coincide. We call $\vartheta$ admissible if all its contours are pairwise disjoint (and different consequently).

Let $\vartheta=\left(\Gamma_{1}, \ldots, \Gamma_{s}\right)$ admissible. We denote $\varrho_{\vartheta}$ to be probability that $\Gamma_{1}, \ldots, \Gamma_{s}$ are present in configuration.

Correlation equations of Minlos-Sinai [9] are

$$
\begin{aligned}
& \varrho(\vartheta)=e^{-\beta|\Gamma(\vartheta)|} \sum_{\bar{\vartheta}}(-1)^{|\bar{\vartheta}|} \varrho([\vartheta-\Gamma(\vartheta)] \cup \bar{\vartheta}), \\
& \varrho(\emptyset)=1 .
\end{aligned}
$$

We fixed for each admissible $\vartheta$ some contour $\Gamma=\Gamma(\vartheta) \in \vartheta$ such that $|\Gamma(\vartheta)|$ (the length of $\Gamma$ ) is not less than $\left|\Gamma^{\prime}\right|$ for any $\Gamma^{\prime} \in \vartheta$ and otherwise arbitrary.

The summation in (2) is through all $\bar{\vartheta}$ (including empty one) such that

1. each $\bar{\Gamma} \in \bar{\vartheta}$ intersects $\Gamma(\vartheta)$;

2. collection $\vartheta^{0} \cup \bar{\vartheta}$ is admissible where $\vartheta^{0}=\vartheta-\Gamma(\vartheta)$.

We shall seek explicit solution of (2): For this reason we consider all sequences

$$
\gamma=\left(\vartheta_{1}, \ldots, \vartheta_{n}\right) \equiv\left(\vartheta_{1}(\gamma), \ldots, \vartheta_{n}(\gamma)\right)
$$

of admissible collections of contours. We require that $\vartheta_{n}=\emptyset, \vartheta_{i} \neq \emptyset, i=1, \ldots, n-1$ and

$$
\vartheta_{j}(\gamma) \supseteqq \vartheta_{j-1}^{0}(\gamma), \quad j=2, \ldots, n
$$

and that each $\Gamma \in \vartheta_{j}-\vartheta_{j-1}^{0}$ intersects with $\Gamma\left(\vartheta_{j-1}(\gamma)\right)$.

Let us put

$$
\begin{aligned}
& a_{\gamma}=\varepsilon_{\gamma} \exp \left[-\beta \sum_{j=1}^{n-1}\left|\Gamma\left(\vartheta_{j}\right)\right|\right] \\
& \varepsilon_{\gamma}=(-1)^{\sum_{j=1}^{n-1}\left|\vartheta_{j+1}-\vartheta_{j}^{0}\right|} .
\end{aligned}
$$

\section{Lemma 1.}

$$
\varrho(\vartheta)=\sum_{\gamma} a_{\gamma}
$$

where the summation is through all $\gamma$ such that $\vartheta_{1}(\gamma)=\vartheta$. Moreover series

$$
\sum_{\gamma}\left|a_{\gamma}\right|
$$

is convergent.

Proof. We prove first absolute convergence. To each $\gamma$ corresponds a sequence of pairs of numbers

$$
r(\gamma)=\left[\left(r_{1}^{1}, r_{2}^{1}\right), \ldots,\left(r_{1}^{n}, r_{2}^{n}\right)\right]
$$


where

$$
r_{j}^{1}=\left|\Gamma\left(\vartheta_{j}\right)\right| \text { and } r_{j}^{2}
$$

is equal to the sum of the lengths of all $\Gamma \in \vartheta_{j+1} \backslash \vartheta_{j}^{0}$.

The number of $\gamma$ such that $r(\gamma)=r$ for given sequence $r$ does not exceed

$$
C^{j=1} \sum_{1}^{n-1}\left(r_{1}^{j}+r_{2}^{j}\right)
$$

From this and from (5) it follows that

$$
\sum_{\gamma}\left|a_{\gamma}\right| \leqq \sum_{r}(C \exp (-\beta)) \sum_{j=-1}^{n-1}\left(r_{1}^{j}+r_{2}^{j}\right) .
$$

In fact the collection $\left\{\Gamma\left(\vartheta_{j}\right): j=1, \ldots, n-1\right\}$ up to permutation coincides with collection

$$
\vartheta_{1} \cup\left(\vartheta_{2} \backslash \vartheta_{1}^{0}\right) \cup \ldots \cup\left(\vartheta_{n-1} \backslash \vartheta_{n-2}^{0}\right) .
$$

Series (8) evidently converges.

Put

$$
\tilde{\vartheta}=\bigcup_{\Gamma \in \vartheta} \Gamma
$$

and for each $R \subset\left(\mathbb{Z}^{v}\right)^{*}$ (dual lattice) put

$$
b_{R}=\sum^{R} a_{\gamma}
$$

where the summation in $\sum^{R}$ is through all $\gamma$ such that

$$
\bigcup_{\vartheta \in \gamma} \tilde{\vartheta}=R
$$

and put $b_{R}=0$ if there is no $\gamma$, satisfying (10).

Theorem 1. For each $\psi_{\vartheta}$ there exists a cluster expansion $\left[\right.$ in $\left(\mathbb{Z}^{v}\right)^{*}$ instead of $\mathbb{Z}^{v}$ and with respect to $\mathfrak{U}=\left\{\right.$ all lines of $\left.\left.\left(\mathbb{Z}^{v}\right)^{*}\right\}\right]$

$$
\psi_{\vartheta}=\sum b_{R}
$$

with

$$
\left|b_{R}\right| \leqq\left(C e^{-\beta}\right)^{|R|}
$$

We denoted $\psi_{\vartheta}$ here a random variable equal to 1 if all $\Gamma \in \vartheta$ are present in configuration and equal to 0 otherwise.

\section{Corollary 1.}

$$
\left|\left\langle\psi_{\vartheta_{1}}, \ldots, \neq \psi_{\vartheta_{n}}\right\rangle\right| \leqq\left(C e^{-\beta}\right)^{\delta\left(\vartheta_{1}, \ldots, \vartheta_{n}\right)} \prod_{i} u_{i} C^{d_{\tilde{\vartheta}_{i}}}
$$

where $\delta\left(\vartheta_{1}, \ldots, \vartheta_{n}\right)$ is minimal $|R|$ among $R$ which contain all $\bar{\vartheta}_{i}$ and is a union of contours. 
Contrary to this we shall prove now

Proposition 2. There is no strong cluster estimates for $\sigma_{t}$ e.g. there is no $\lambda=(\lambda(\beta))>0$ such that $\lambda(\beta) \rightarrow 0$ if $\beta \rightarrow \infty$ and

$$
\left|\left\langle\sigma_{t_{1}}, \ldots, \sigma_{t_{n}}\right\rangle\right| \leqq n_{1} ! \ldots n_{m} ! \lambda^{d_{\left.t_{1}, \ldots, t_{n}\right\}}}
$$

where $m$ is the number of groups of identical $t_{i}$ and $n_{j}$ - the number of elements in these groups.

Proof. Estimate (13) being valid one can consider Gibbs measure with density

$$
\frac{d \mu_{V, h}}{d \mu_{+}}=Z_{V, h}^{-1} \exp \left(h \sum_{t \in V} \sigma_{t}\right)
$$

where $\mu_{+}$is Gibbs measure in an infinite volume - a $(+)$-pure phase.

It follows that limit measure $\mu_{h}=\lim _{V} \mu_{V, h}$ is translation invariant and has momentae $\langle\psi\rangle_{h}$ given by a series in semiinvariants $\left\langle\sigma_{t_{1}}, \ldots, \sigma_{t_{n}}\right\rangle$ which converges and is analytic in $h$ for $|h|$ sufficiently small because of (13). But this contradicts to the known fact [10] that for $h \neq 0$ translation invariant measure having correspondent Gibbs conditional probabilities is unique and is not analytique in the point $h=0$.

\section{References}

1. Glimm, J., Jaffe, A., Spencer, T.: Ann. Math. 100, 585-632 (1974)

2. Malyshev, V.A.: Complete cluster expansion for weakly coupled Gibbs random field. In: Manycomponent random systems. Berlin, Heidelberg, New York: Springer 1978

3. Duneau, M., Jagolnitzer, D., Souillard. B.: Commun. math. Phys. 40, 83-95 (1975)

4. Duneau, M., Jagolnitzer, D., Souillard, B.: Commun. math. Phys. 31, 191-208 (1973)

5. Sylvester, G.S.: Weakly coupled Gibbs measures. Rockefeller Univ. Preprint 1977

6. Rota, J.-C.: Z. Wahrscheinlichkeitstheorie verw. Gebiete 2, 340-368 (1964)

7. Malyshev, V.A.: Perturbations of Gibbs random fields. Mnogokomponentnye sluchayn. system. Moscow: Nauka 1978

8. Glimm, J., Jaffe, A., Spencer, T. : In: Lect. Notes Phys. 25, 132-242 (1973)

9. Minlos, R. A., Sinai, Ya.G.: Trudy Moskov. Mat. Obsc. 19, 121-196 (1968)

10. Ruelle, D.: Ann. Phys. 69, 364-374 (1972)

11. Birkgoff, G. : Lattice theory. New York: 1948

Communicated by A. Jaffe

Received May 16, 1978 
